\title{
Comparison between Optical Fiber Amplifiers of Information Signals
}

\author{
Mohammed Yousef AL-Gawagzeh. \\ Department of Electrical power Engineering, Faculty of Engineering \\ Al-Balqa Applied University \\ JORDAN
}

Received: October 10, 2020. Revised: November 13, 2020. Accepted: November 16, 2020. Published: November 16, 2020.

\begin{abstract}
This research is devoted for reviewing a different types of fiber optical amplifiers for construction the optical system of transmission such erbium-doped fiber amplifier (EDFA), Thulium-doped fiber amplifier (TDFA), Compelled Combinational Dispersion (CCD), their principles of work advantages, and disadvantages, with purpose of increasing $₫ h$ pass band spectrum and to decrease some disadvantages such attenuation and dispersion. also considered a suggested method of simultaneous stokes and anti-stokes strengthening of compelled combinational dispersion in fibers.
\end{abstract}

Keywords-Amplifiers, fiber optic, signal, transmission.

\section{INTRODUCTION.}

O transmit information signals for long distances (more than $100 \mathrm{~km}$ ), it is necessary to decrease the losses (attenuation dispersion) in the fiber optic cables. The most important points for any optical amplifier are the noise figure and the gain. Owing to dispersion and absorption, which weakening the information signals in the process of transfer over fiber communication lines for long distances, it is necessary to use.

consistently located Amplifiers (repeaters).

Optical amplifiers based on laser principle, they received the optical signals and regenerates all wavelengths. Two types of completely optical amplifiers recently have been used on wide scale. such optical repeaters are based on the phenomenon of compelled issue in fibers which activated by earth metals ions, and Raman amplifiers in which the nonlinear optical effect of compelled combinational dispersion (CCD) in fibers is used. Such systems strengthen optical signals relatively in wide range of frequencies including tens and hundreds of information channels related to waves lengths. They are not sensitive to speed of data transmission down to $100 \mathrm{~Gb} / \mathrm{s}$ and works with any formats of data transmission [1-16].

\section{MATERIALS AND DISCUSSION}

\section{A. ERBIUM-DOPED FIBER AMPLIFIERS (EDFA).}

Now mostly used optical Amplifiers are the Amplifiers which activated by erbium ions (erbium-doped fiber amplifier -
EDFA) and allowing effectively to strengthen the signals with least absorption of quartz glass in a spectral strip 1535,1565 nanometers, and also in the field of $1570 \ldots 1610$ nanometers [4].

In communication lines three various types of erbium fiber amplifiers (EDFA) are applied [4, 5]. first type is the power amplifier which established directly after Multiplexer and intended for strengthening the broadband signals up to a level which cannot be reached in separate semiconductor lasers, strengthening here reaches $30 \mathrm{~dB}$.the second type is the linear Amplifier with the purpose of signal indemnification which occurs due to attenuation in optical fiber, strengthening in linear Amplifier usually makes $18-23 \mathrm{~dB}$. Third type is the preliminary amplifier which established directly ahead of the DE multiplexer and provides an increase in attitude of $\mathrm{S} / \mathrm{N}$ on the input of electronic cascades of strengthening after optoelectronic detectors system and strengthening here makes 18$23 \mathrm{~dB}$. (EDFA) types structurally differ by the levels of bump capacity, activator ions concent " ' iber length. the scheme of simple single cascade 1 Erbium own on fig.1 [4, 5]. fiber

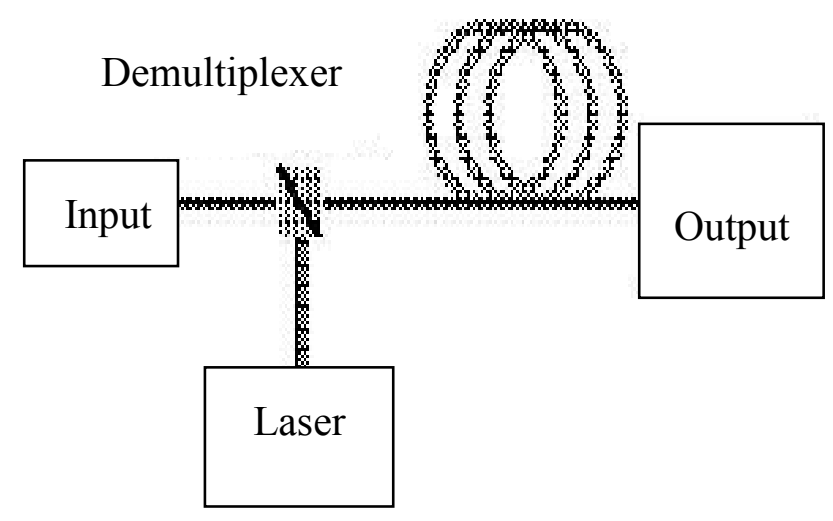

Fig.1: The basic scheme of single cascade erbium fiber amnlifier

Optical fiber bump is carried out on wave's lengths of 980 nanometers or 1480 nanometers, and then laser radiation with 
help of optical multiplexer is entered into an intensifying fiber. Energy bump is absorbed by erbium ions which activated by quartz fiber and translating these ions in the raised condition. Strengthening occurs, when the alarm photon with a wavelength of 1550 nanometers initiates transition of the raised erbium ion in the basic condition with radiation of an identical photon.

The single cascade amplifier usually contains one or two bump lasers $[9,10]$, thus for strengthening, increasing a polarizing or frequency combination of bump sources is carried out.

The simple single cascade amplifier does not satisfy all requirements of modern telecommunication systems; therefore, it supplement with a number of special devices. For prevention of bump radiation hit on the input or output of the amplifier it is necessary to use optical insulators. For reception of the capacity information and a signal spectrum on the input and output of the amplifier optical a splitter with measuring devices are used. Signals of these devices are used for controlling the capacity of laser radiation bump, and control of strengthening factor, and for notification about an emergency $[4,5]$.

By transfer a with huge number of information channels which multiplexing by waves length, the expanded range of frequencies in which it is complex to provide uniform frequency characteristic (EDFA) is used. Frequency dependence becomes an acute problem in these systems. this specified disadvantage is eliminated by using a smoothing narrow band optical filters for easing the signals on frequencies which corresponds greater amplification coefficients. Smoothing the frequency characteristic of strengthening is usually reached at maintenance of demanded values of some parameters, such as input power levels of signal and bump, fiber length, etc. In single cascade amplifiers, the smoothing of frequency characteristic led to loss of output power which can reach $10 \mathrm{~dB}$ or more, or to additional easing of the information signal before its strengthening. Therefore, multi-cascade schemes are used for smoothing amplifiers [4, 5].

The optical multi-cascade (EDFA) scheme is illustrated on fig. 2.

\section{First Second cascade cascade \\ EDFA EDFA}

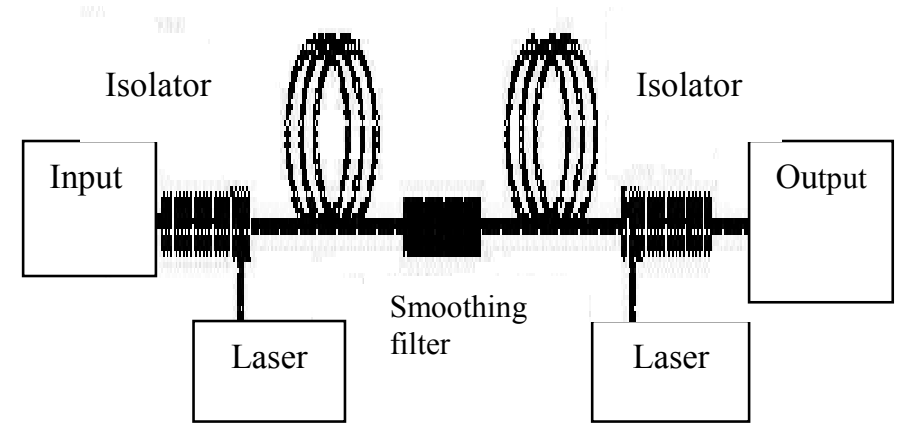

Fig.2: Multicascade scheme of erbium amplifier multiplexing by of waves lengths.
As a rule, in the first cascade used a passing laser with wave length 980 nanometer, and an opposing laser with wave length 1480 nanometer in the second cascade and this allow us to avoid laser radiation hit on the input and output of information network.

The smoothing filter lead to an essential loss in multicascade (EDFA), therefore these filters must be founded between cascades of strengthening.

From strengthening the spectral curve of multicascade (EDFA) which illustrated on figure 3, which show that application of the smoothing filter leads to non-uniformity of strengthening less than $0.3 \mathrm{~dB}$ in all frequency strip.

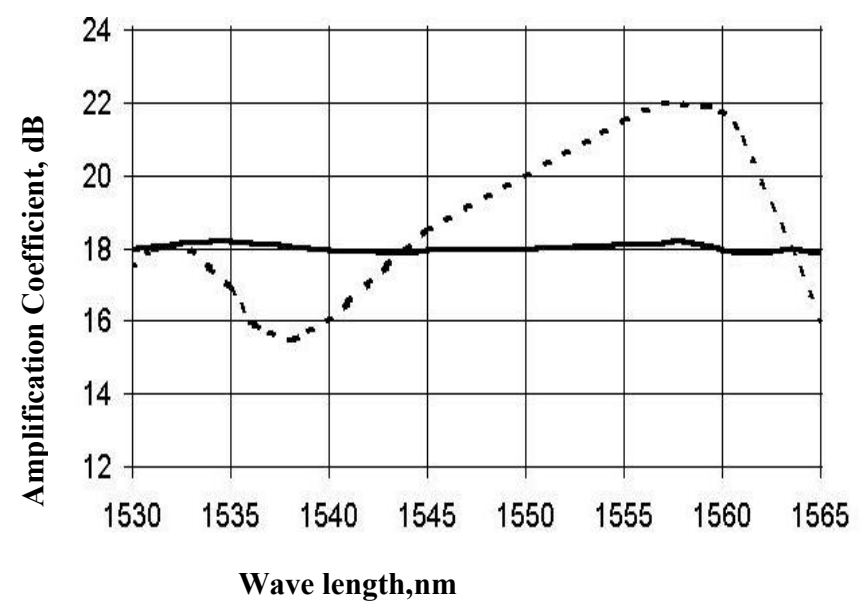

_. _. Without smoothing filter,____ with smoothing filter

Fig.3: strengthening spectral curve of multicascade erbium amplifier.

Till now the (EDFA) are the basic kind of optical amplifiers for data transmission over optical fiber communication lines for long distances [4]. And for increasing the data transmission speed and quantity of spectral channels, we need an occurrence of alternative technologies.

\section{B. THULIUM-DOPED FIBER AMPLIFIERS (TDFA).}

Thulium-doped fiber amplifier (TDFA) work similarly as (EDFA), where the optical signal strengthening occurs due to transformation the energy bump into energy signals during radiation distribution in intensifying fiber $[6,7]$. The difference between (EDFA) and (TDFA) consists in the way of strengthening which defined by ions activators and bump scheme. The principal scheme of (TDFA) is illustrated on figure 4. 


\section{Thulium fiber}

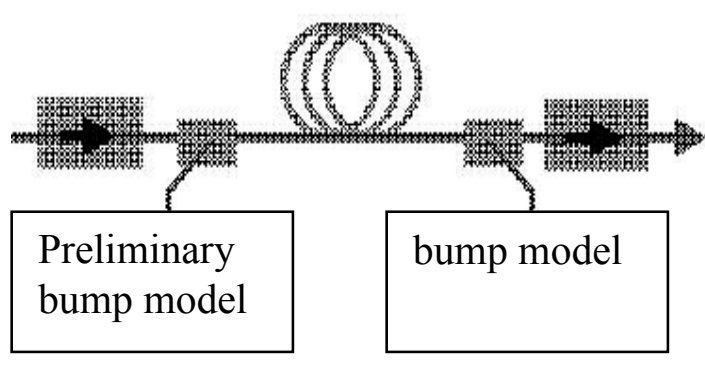

Fig.4: The basic thulium-doped fiber amplifier scheme

The power levels scheme on figure.5

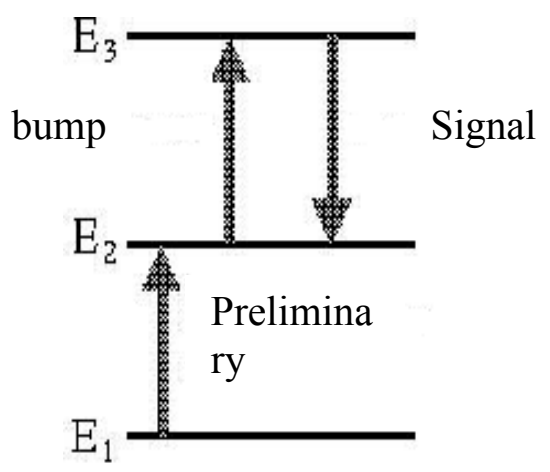

Fig.5: power levels scheme of thulium-doped fiber amplifier

illustrates process of optical strengthening in (TDFA), where Strengthening is carried out using two bump waves with identical or various frequencies. Photons of preliminary bump translate activator ions on the power level (E2) that being the bottom operating level, then photons of basic bump are used for settling the top working power level (E3), the compelled transition which on level (E2) provides strengthening of optical signals.

Using this specified bump scheme, the strengthening center of multi component quartz fiber with high concentration of thulium ions is near to wavelength of 1460 nanometers (fig. 6).

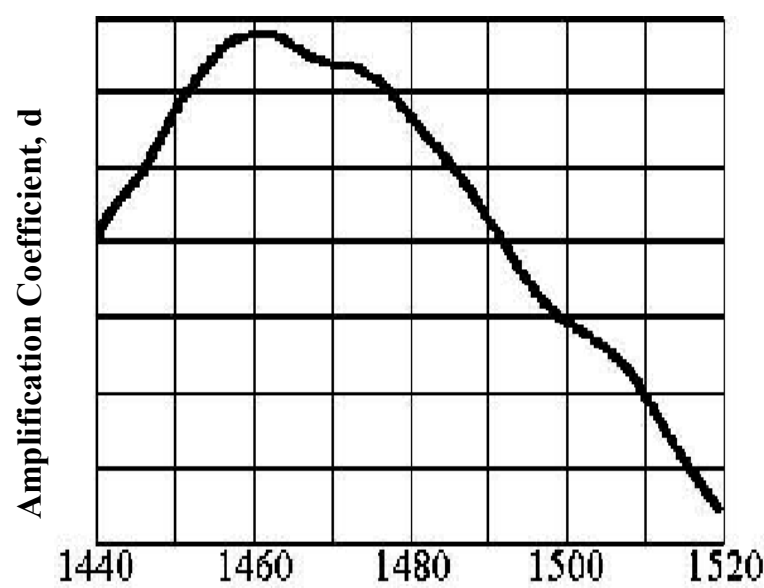

Fig.6: Strengthening spectral curve of thulium-doped fiber amplifier.

The width of a spectral strip of strengthening with a level $20 \mathrm{~dB}$ makes in such fibers about 35 nanometers, and the maximum strengthening can reach $31 \mathrm{~dB}[6,7]$. Applying other bump schemes is possible to receive a strengthening in a range of lengths of waves $1480 \ldots 1510$ nanometers [6].

The basic obstacle for wide circulation of (TDFA) is insufficiently high durability of an optical fiber amplifier. Other problem is the maintenance complexity high quality of optical connection due to physical and chemical structure of fiber. Besides the structure of a fiber which includes zirconium that causes the high manufacture price cost which means a high cost of (TDFA), so these reasons minimize their wide circulation.

\section{COMPELLED COMBINATIONAL DISPERSION OF FIBER AMPLIFIERS (CCD).}

The systems which constructed based on (EDFA) and (TDFA) possess a greater noise level on frequencies of information signals which caused by influence of spontaneous ions radiations of activators. Strengthening based on (CCD) allows providing additional increase of signal level without essential noise increasing at signal frequency [7].

Strengthening during (CCD) is caused by nonlinear optical swapping of bump energy into stocks energy. for example, for achievement of information signal strengthening on wave length of 1550 nanometers, it is necessary to use bump energy on a wave length of 1455 nanometers, thus due to wide strip of (CCD) of quartz fiber the strengthening occurs on the adjacent frequencies (fig. 7) [7, 8]. 


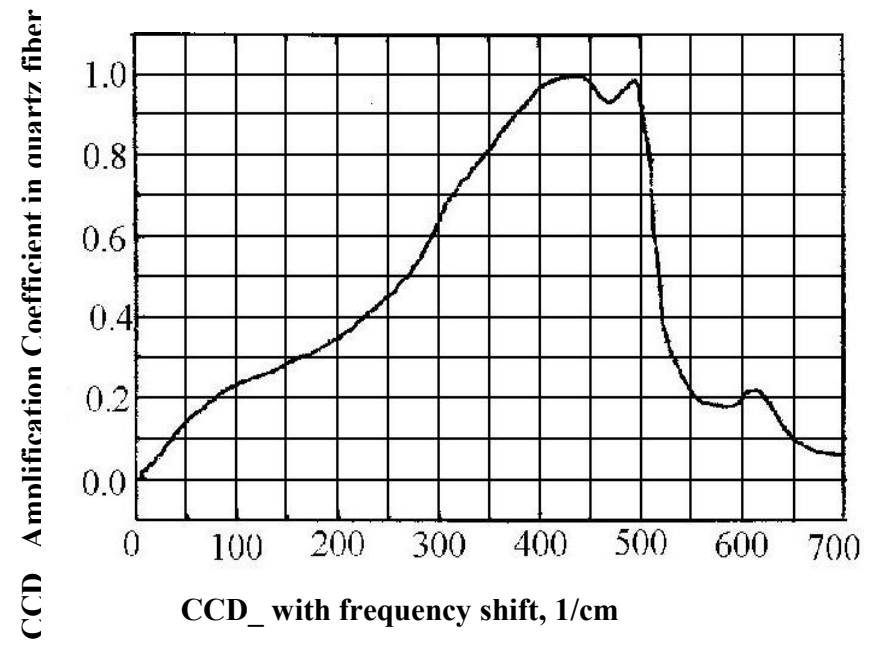

Fig.7: Dependence of compelled combinational dispersion Strengthening coefficient in quartz fiber on frequencies shift.

Process of $(\mathrm{CCD})$ strengthening occurs in any optical fiber with corresponding energy bump and consequently it can be used for parameters improvement not only new, but also already existing in optical lines of information transfer. For smoothing the strengthening curve and for maintenance a greater amplifier capacity we can use the energy bump on several waves' lengths (fig. 8) [7,8].

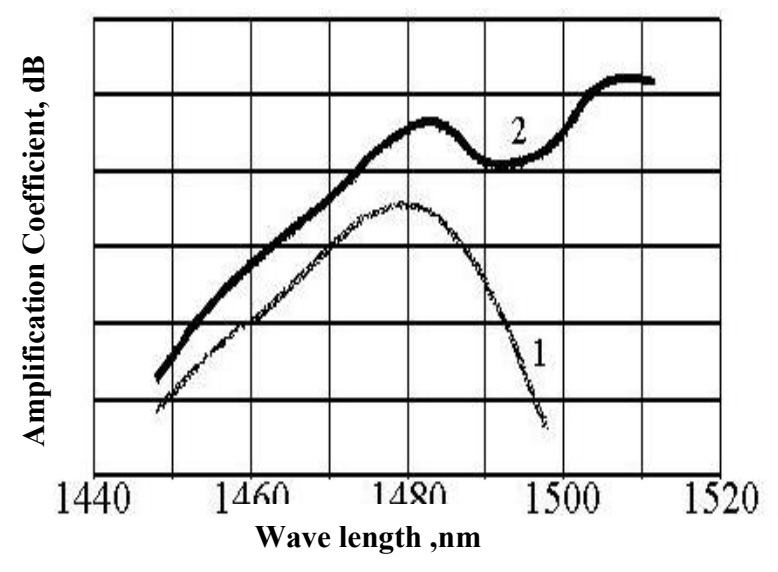

Fig.8: Spectral curves strengthening of (CCD) amplifiers:

1) Energy bump at one wave length

2) Energy bump at tow waves lengths
The basic disadvantage of (CCD) amplifiers is their low efficiency in comparison with (EDFA). To achieve a strengthening of $30 \mathrm{~dB}$ for (EDFA) the needed energy bump is nearby $50 \mathrm{~mW}$, whereas for similar strengthening of (CCD) amplifier the necessary capacity is more than $1 \mathrm{~W}$ [7].

Recently a powerful lasers diode with capacity more than 10 $\mathrm{W}$ have appeared [7], which allowed to create an effective (CCD) amplifier and capable to compete with (EDFA). On this basis a fast occurrence of commercial (CCD) amplifiers is expected.

The (CCD) amplifiers can be used for strengthening in a Sband $(1480 \ldots 1520$ nanometers) and in a L-band $(1570 \ldots 1610$ nanometers) and in a range from 1642 up to 1672 nanometers $[7,8]$. In optical networks the (CCD) can be used as a single mechanism of strengthening or for compensation with (EDFA).

\section{HYBRID FIBER AMPLIFIERS.}

Optical amplifiers cannot satisfy all needs of modern telecommunication systems, therefore development of new kinds of optical amplifiers now are actively conducted. owing to the strengthening band of (CCD)amplifiers can be shifted aside higher or lower frequencies comparing to a strengthening band of (EDFA)and if we use an enough powerful bump lasers, so it is possible to coordinate amplifiers frequency bands of strengthening to be much wider than at each of amplifiers separately. Thus, it is considerably possible to smooth the nonuniformity of strengthening curves of separate amplifiers [9, 10] (fig. 9).

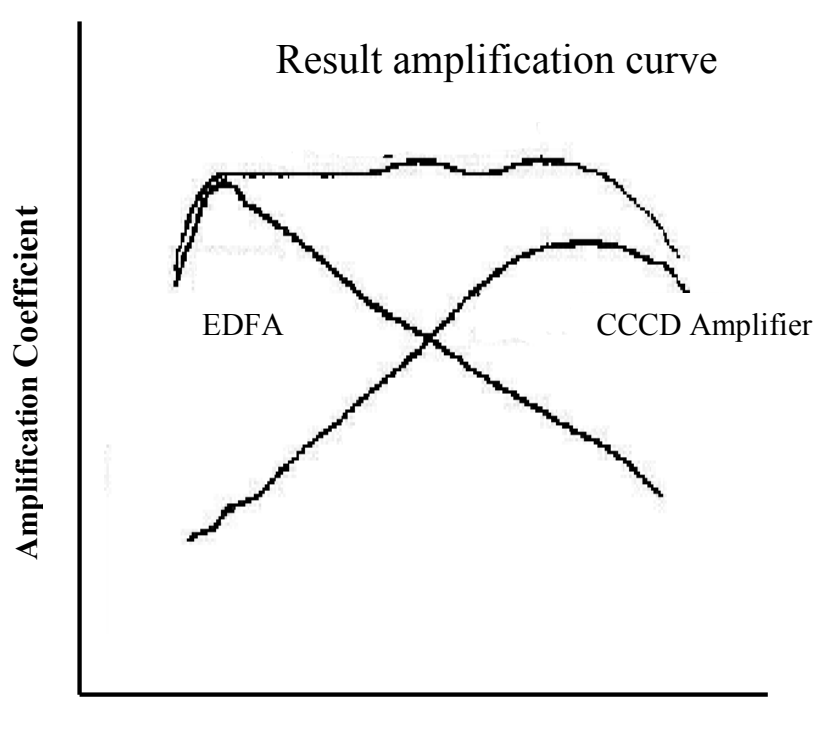

Fig.9: Smoothing curves of strengthening for (CCD) and (EDFA) amplifiers. 


\section{E. SUGGESTED STOCKS AND ANTI-STOCKS OF (CCD) AMPLIFIERS.}

For simultaneous strengthening in quartz fiber transparency windows of (1310 and 1550 nanometers) it is possible to use a combined Stocks and Anti-stocks (CCD) strengthening with conditions of phase quasi-synchronism [11,12]. And for maintenance the condition of phase quasi-synchronism, the fiber should consist of alternating areas with nonlinear properties (active layers) and without nonlinear properties (passive layers).

The basic properties of (CCD) phase quasi-synchronism in detail have been investigated in [13]. With use of the given technique, the calculations have shown that the fiber length on which strengthening can reaches $13 \mathrm{~dB}$.

Due to a wide frequency band of a quartz fiber, the (CCD) strengthening occurs not on one frequency but in some other frequencies bands (fig. 10) [11, 12]

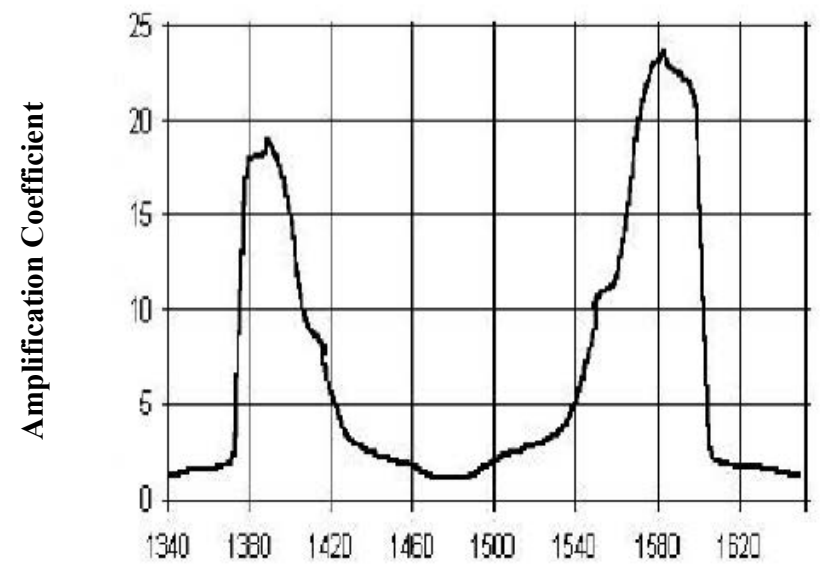

Fig.10: Spectral curve of Stocks and Anti-stocks strengthening in auartz fiber.

As in a wide range of bump wavelength of $(1400 \ldots 1500$ nanometers) the layered structure remains constant, for increasing the capacity of amplifier or increasing and smoothing the strengthening band, we can use the energy bump on several waves lengths.

The basic disadvantage of the given method is high complexity of realization of the layered structure, which consisting of big number of layers.

\section{CONCLUSION}

Now in fiber optical communication lines used completely Erbium amplifiers, take in consideration that their requirements satisfaction opportunities for next generation of telecommunication systems are not enough. For increase the pass band (number of spectral channels) and for smoothing the strengthening curve it is necessary to use a hybrid amplifier. Realization of the hybrid amplifier with simultaneous stocks and anti-stocks strengthening will allow increasing a pass band. The growth of data transmission speeds compared with low speed of Erbium amplifiers will cause several problems, therefore CCD amplifiers will take the lead positions in the market of fiber optical amplifiers.

The future works are expected to concentrate on the reducing of noise figure with high pump power.

\section{REFERENCES:}

[1] K. Nakagawa, S. Nishi, E. Yoneda,"Trunk and distribution network application of erbium-doped fiber amplifier" $J$. Lightwave Technol. V. 9, pp. 198-207. 2005.

[2] G. Randy, L. I. Tingyc, "Optical amplifiers transform long distance light voice telecommunications", Proc. IEEE, vol 84, pp. 870-883, 2000.

[3] P. Urquhart, 'Review. Of. Rare earth doped fiber lasers and amplifiers', IEE Proc. J, vol 135, pp. 385-407. 2003.

[4] D. A Trivedi., T. Strite ,"EDFAs pump up the power", WDM solutions, vol 4. pp. 14-20,2000.

[5] D. C. McCarthy," Characterization of erbium-doped fibers and application to modeling 980-nm and 1480-nm pumped amplifiers ", Photonics Spectra. vol 7. Pp. 88-98. 2001.

[6] C.P.Yvonne," Interferometer provides tuning and Filtering for DWDM ," WDM solutions 7. pp. 9-20. 2010.

[7] M. Islam., M. Nietubyc," Optical amplification using polarization diversity pumping," WDM solutions ,vol 3, pp 53-62. 2009.

[8] J. Hecht,"Optical amplifiers revolutionize communications," Laser Focus World, pp. 28-32. Sep. 1998.

[9] L. Kristin," Multistage amplifier provides gain across $80 \mathrm{~nm}$," Laser Focus World, pp. 22-24. Sep. 2008.

[10] J. Hecht., "All-optical converters promise improved networks,"Laser Focus World, pp. 159-164. Apr. 2001.

[11] K. E. Stubkjaer," Semiconductor Optical Amplifier-Based AllOptical Gates for High-Speed Optical Processing, "IEEE Journal on Selected Topics in Quantum Electronics, vol. 6, No. 6, pp. 1428-1435. Nov./Dec. 2000.

[12] Zhao et al,"All-Optical 2R Regeneration Based on Polarization Rotation in a Linear Optical Amplifier," IEEE Photonics Technology Letters, vol. 15, No. 2, pp. 305-307. Feb. 2003. 
[13] Maeda et al,"Optical Amplifiers," IEEE Photonics Technology Letters, vol. 15, No. 2. pp. 257-259. 2003.

[14] N. M. Ibrahim,A. Abubaker," A Comparision of optical Amplifiers in optical communication systems EDFA, SOA and RAMAN," International Journal of Current Research, Vol. 6, Issue, 09, pp.8738-8741, September 2014.

[15] B. Utreja, H. Singh," A review paper on comparison of optical amplifiers in optical communication systems," Canadian Journal on Electrical and Electronics Engineering, Vol. 2, No. 11, November 2011.

[16] A. Rani, S. Dewra," Semiconductor optical amplifiers in optical Communication system-Review," International Journal of Engineering Research \& Technology (IJERT), Vol. 2 Issue 10, pp.271-2719, October - 2013.

\section{Creative Commons Attribution License 4.0} (Attribution 4.0 International, CC BY 4.0)

This article is published under the terms of the Creative Commons Attribution License 4.0

https://creativecommons.org/licenses/by/4.0/deed.en_US 